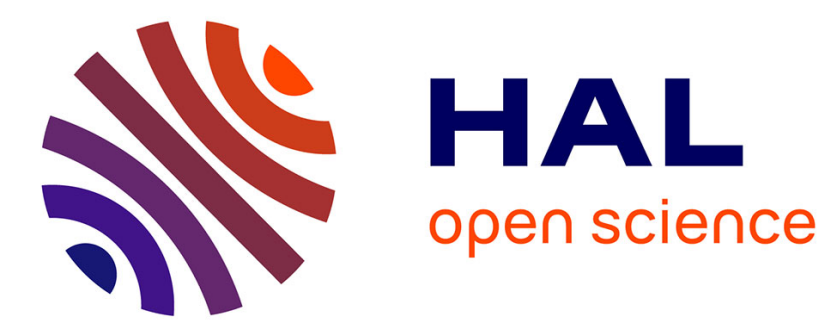

\title{
Les contrôles de filiation dans les populations
}

D. François, Marie-Yvonne Boscher, J. C. Mériaux, T.C. Nguyen

\section{- To cite this version:}

D. François, Marie-Yvonne Boscher, J. C. Mériaux, T.C. Nguyen. Les contrôles de filiation dans les populations. Productions Animales, 1992, hs (hs), pp.273-276. hal-00896030

\section{HAL Id: hal-00896030 \\ https://hal.science/hal-00896030}

Submitted on 1 Jan 1992

HAL is a multi-disciplinary open access archive for the deposit and dissemination of scientific research documents, whether they are published or not. The documents may come from teaching and research institutions in France or abroad, or from public or private research centers.
L'archive ouverte pluridisciplinaire HAL, est destinée au dépôt et à la diffusion de documents scientifiques de niveau recherche, publiés ou non, émanant des établissements d'enseignement et de recherche français ou étrangers, des laboratoires publics ou privés. 
D. FRANCOIS, Marie-Yvonne BOSCHER, J.C. MÉRIAUX, T.C. NGUYEN

INRA Laboratoire d'Analyses des Groupes Sanguins 78352 Jouy-en-Josas Cedex
Apports actuels et futurs des

marqueurs génétiques dans

l'amélioration des populations

animales

\section{Les contrôles de filiation dans les populations}

Résumé. Le contrôle de filiation est utilisé par les organismes en charge de l'amélioration génétique des espèces d'élevage. Il permet de contrôler l'exactitude des généalogies enregistrées dans les fichiers des chaînes nationales d'identification. Il est réalisé en comparant les hémotypes des trois membres (père, mère, produit) des familles contrôlées. Les analyses comportent un grand nombre de tests immunologiques et biochimiques conférant au contrôle de filiation une grande efficacité dans la détection des familles erronées.

L'enregistrement des généalogies ou filiations des animaux a débuté au siècle dernier avec les premiers Livres Généalogiques (herd-books bovins, flock-books ovins et stud-books équins). La loi sur l'élevage de 1966 a organisé l'identification sous forme numérique des animaux et la circulation de ces informations d'état-civil entre les fichiers zootechniques départementaux, régionaux, nationaux et raciaux.

Les recherches sur les groupes sanguins des animaux ont été initiées à l'Institut Pasteur dans les années cinquante (Dujarric de la Rivière) et développées à l'INRA, à Jouy, dans les années soixante (Grosclaude). La méthode du contrôle des filiations par l'analyse des groupes sanguins et l'étude de leur hérédité a été mise en oeuvre au Laboratoire d'Analyse des Groupes Sanguins d'abord chez les bovins : premières analyses de taureaux d'Insémination Artificielle (IA) en 1958 ; puis chez les ovins (1972), les équins (1975) et plus récemment les caprins (1990).

\section{1 / Utilisation des contrôles de filiation (CF)}

Les différents acteurs de l'amélioration génétique utilisent le CF pour s'assurer de l'exactitude des généalogies enregistrées dans les fichiers des chaînes nationales d'identification. L'importance de cette exactitude s'est encore accrue avec l'utilisation récente du modèle animal dans l'évaluation des reproducteurs.

\section{1 / Règlementations édictées par le Ministère de l'Agriculture}

Le Ministère impose des CF pour les reproducteurs à large diffusion tels que les taureaux d'IA (arrêtés du $17 / 02 / 59$ et du $08 / 03 / 74$ ) ou les étalons de monte publique (arrêté du 29/12/76). Le contrôle obligatoire a également été institué pour tous les veaux nés de transfert d'embryon : Note de Service (N.S.) Direction de la Production et des Echanges, Ministère de l'Agriculture $01 / 07 / 87$; pour tous les équidés produits d'IA (arrêté du 23/12/86) ainsi que pour tous les produits de race Pur-sang (N.S. Haras Nationaux 1986) et de race Arabe (N.S. Haras Nationaux 1988).

\section{2 / Le contrôle de filiation dans la base de sélection}

\section{a / Unités de sélection}

La règlementation ministérielle pour les taureaux d'IA s'applique au moment de l'agrément. Les unités de sélection utilisent le CF dès l'entrée en station. Un nombre beaucoup plus important d'animaux est donc ainsi contrôlé. De plus certains protocoles de testage sur descendance en races allaitantes assortissent les mesures sur descendants de contrôles de filiation afin d'assurer l'efficacité de l'évaluation génétique. En espèce ovine et caprine, les analyses (contingentées) s'adressent en priorité aux jeunes béliers et boucs testés par les unités de sélection.

\section{b / Livres généalogiques}

Clients majoritaires du Laboratoire jusqu'au milieu des années 80, les Livres Généalogiques conduisent de vastes programmes de CF. Le Herd-Book Charolais vérifie ainsi chaque année 3000 filiations.

Chez les équidés, le Service des Haras Nationaux contrôle, par sondage, les enregistrements effectués dans chacune des races de chevaux de sang. Ce système de contrôle ( 1 filiation sur 5 ) est en voie d'être supplanté par le contrôle systématique (voir 1.1).

Dans l'espèce ovine, l'Institut de l'Elevage attribue annuellement une partie du contingent d'analyses aux UPRA. 


\section{3 / Le contrôle de filiation au niveau des élevages de production}

\section{a / Vérifications d'identification par sondage}

L'Institut de l'Elevage conduit des programmes de contrôle par sondage de l'identification réalisée par les Etablissements Départementaux de l'Elevage (EDE). Le programme bovin touche ainsi 15 départements par an, 50 élevages par départements, 4 filiations (déclarées dans l'année) par élevage soit 3000 veaux et environ 6000 analyses (la plupart des pères étant déjà analysés par le laboratoire).

\section{b / Contrôle de filiation à l'enregistrement de la naissance}

Les enregistrements des déclarations de naissance des veaux par les EDE ou les syndicats de contrôle de performances obéissent à certaines conditions. Lorsque ces conditions ne sont pas remplies (durée de gestation anormale, délai de déclaration non respecté...) ou que l'éleveur (ou l'agent identificateur) n'est pas sûr de la filiation (inséminations multiples, naissances simultanées...), l'EDE commande le CF avant de valider la déclaration de naissance.

Peuvent être rangés dans cette rubrique, les CF des veaux nés de transfert d'embryon pour lesquels un premier enregistrement sous réserve du résultat du CF est effectué, puis validé par le résultat positif du CF.

Chez les équidés où les enregistrements de double paternité sont autorisés, les contrôles de ces filiations particulières sont faits a posteriori et entraînent ensuite une rectification de l'enregistrement à l'édition du livret signalétique du poulain.

Notons également qu'en cas de doute sur l'identité d'un animal, un contrôle de son hémotype peut être pratiqué en comparaison avec une analyse effectuée dans le jeune âge de l'animal. Ce type de contrôle est mis en oeuvre par le Service des Haras lorsqu'il y a un doute sur l'identité d'un cheval lors de la validation de son livret signalétique (compétition, reproduction).

\section{2 / L'analyse des groupes sanguins}

Les maîtres d'oeuvre des contrôles de filiation nous adressent des prélèvements sanguins (tube de $5 \mathrm{ml}$ avec anticoagulant) des animaux dont le groupe sanguin n'est pas encore connu. L'analyse sera plus ou moins complexe selon l'espèce (tableau 1) en fonction

Tableau 1. Techniques d'analyse des groupes sanguins en fonction de l'espèce étudiée.

\begin{tabular}{|l|cc|c|c|}
\hline & \multicolumn{2}{|c|}{$\begin{array}{c}\text { Groupes érythrocytaires } \\
\text { Hémolyse }\end{array}$} & $\begin{array}{c}\text { Electrophorèse } \\
\text { de protéines }\end{array}$ & $\begin{array}{c}\text { Systèmes } \\
\text { génétiques }\end{array}$ \\
\hline Bovins & 75 & - & - & 11 \\
\hline Equins & 9 & 24 & 10 & 17 \\
\hline Ovins & 30 & 2 & 4 & 12 \\
\hline Caprins & 28 & - & - & 6 \\
\hline
\end{tabular}

du polymorphisme connu (tableau 2) et de l'efficacité recherchée (paragraphe 4).

\section{1 / Quelques définitions}

L'ensemble des caractères antigéniques (groupes érythrocytaires) et biochimiques (variants électrophorétiques) forme l'hémotype de l'animal.

L'étude de familles permet de séparer les caractères liés et les caractères indépendants. Chaque groupe de caractères liés est appelé système génétique et recouvre 1 ou plusieurs loci contigus.

\section{2 / Les groupes érythrocytaires}

Pour l'analyse des groupes érythrocytaires, les échantillons sont centrifugés, lavés. Les globules rouges sont mis en contact avec un volume équivalent d'anticorps pour chacune des spécificités antigéniques à tester. Pour l'hémolyse, un troisième élément appelé complément est ajouté pour révéler la formation du complexe antigène-anticorps.

Les distributions sont réalisées sur des plaques creusées d'alvéoles $(40 \times 40)$ réalisées spécialement pour le laboratoire. Les échantillons sont distribués en ligne et les anticorps en colonne. La réaction est favorisée par des agitations périodiques et une incubation de 3 heures en étuve régulée à $27^{\circ} \mathrm{C}$ (hémolyse).

Une fois révélées, les plaques sont d'une part photographiées, d'autre part saisies par un système de lecture optique pour l'hémolyse et sur une table de digitalisation pour l'agglutination. Les données intègrent ensuite les chaînes informatisées des contrôles de filiation et sont reliées aux données d'état-civil figurant sur les documents accompagnant les prises de sang.

Les anticorps sont produits par le laboratoire dans un atelier de sérologie. Des immunisations sont programmées sur un important troupeau expérimental entièrement dédié à cette activité. Les sérums obtenus après ces immunisations subissent des séparations et purifications successives pour aboutir aux réactifs, spécifiques d'un seul antigène.

\section{3 / L'électrophorèse de protéines}

Parallèlement à ces techniques immunologiques, sont mises en oeuvre des techniques biochimiques permettant de détecter le polymorphisme de protéines sanguines par électrophorèse. Le principe est le suivant : les protéines sont des molécules chargées électriquement et leur charge va leur conférer la capacité de migrer sous l'action d'un champ électrique. Chaque protéine est codée par un gène de structure et chaque variant électrophorétique d'une protéine est sous le contrôle d'une forme allélique de son gène de structure. A la différence des allèles des systèmes d'antigènes érythrocytaires, les allèles contrôlant les variants électrophorétiques sont généralement codominants et un individu hétérozygote présentera donc deux variants différents détectables par électrophorèse. 
Tableau 2. Le polymorphisme des groupes érythrocytaires chez les bovins.

\begin{tabular}{|c|c|c|c|}
\hline Systèmes & Antigènes* & Allèles & Phénotypes \\
\hline A & 2 & 3 allèles & 3 \\
\hline B & 40 & 800 complexes alléliques & très nombreux \\
\hline C & 17 & 120 complexes alléliques & très nombreux \\
\hline S & 8 & 20 complexes alléliques & très nombreux \\
\hline F & 2 & 2 allèles & 3 \\
\hline M & 2 & 3 allèles & 2 \\
\hline L, Z, J, R', T' & 1 & 2 allèles & 4 \\
\hline
\end{tabular}

* ou plus exactement: déterminants antigéniques ou épitopes

Au système $B$ bovin la structure génétique sous-jacente mesure environ 0,7 centi Morgan et correspond sans doute à un groupe de loci. Les systèmes les moins polymorphes correspondent chacun à un locus.

\section{3 / Principe du contrôle de filiation}

\section{1 / Enoncé du principe}

Le contrôle de filiation se base sur la comparaison des hémotypes des animaux de la famille considérée. On y recherche les ressemblances et dissemblances, selon les lois de l'hérédité.

Le résultat rendu est un diagnostic de compatibilité si les lois de la génétique mendélienne sont vérifiées, ou d'incompatibilité si elles ne le sont pas. L'incompatibilité permet d'exclure la famille proposée mais la compatibilité ne permet pas d'en affirmer l'authenticité. En effet, plusieurs parents peuvent avoir des combinaisons d'hémotypes conduisant à des diagnostics de compatibilité. Pour augmenter la probabilité d'exclusion, il faut étudier un maximum de loci, eux-mêmes les plus polymorphes possibles.

\section{2 / Illustration}

La première approche est phénotypique. Le groupe sanguin est présenté comme la liste des caractères révélés par l'analyse. La deuxième approche est génotypique et prend en compte les allèles transmis par chacun des 2 parents proposés.

Le tableau 3 nous illustre l'exemple d'un système sanguin à 2 antigènes $A$ et $B$. Sachant que ce système peut présenter les 4 phénotypes $A, B,-, A B$. Soit, du point de vue génotypique, 1 locus considéré avec 3 allèles possibles $\mathrm{A}, \mathrm{B},-$.

La compatibilité d'une filiation répond au principe suivant : le produit doit avoir reçu un allèle de chacun de ses parents. Il est possible d'en dégager 2 règles principales d'exclusion :
- la première règle d'exclusion de la proposition de filiation est illustrée par la famille 1 :

le produit présente un caractère (B) absent chez les deux parents proposés.

- deuxième règle d'exclusion illustrée par la famille $\mathrm{n}^{\circ} 2$ : la conclusion de cette filiation nécessite la connaissance des génotypes parentaux (étude des groupes sanguins de leurs propres ascendants ou d’autres descendants déjà analysés).

Le génotype paternel A/A entraîne un diagnostic d'incompatibilité car le produit ne présente aucun des 2 allèles de son père.

Ce raisonnement s'applique à tous les systèmes étudiés (voir tableaux 1 et 2 ).

En théorie, aux réserves génétiques près (mutation, recombinaison, allèle nul non identifié) l'incompatibilité sur un seul système suffit à rejeter la proposition de famille. Cela suppose une parfaite connaissance du déterminisme génétique du système utilisé. La compatibilité est exigée sur la totalité des systèmes étudiés.

\section{4 / L'efficacité du contrôle de filiation : le pouvoir d'exclusion}

L'efficacité d'une méthode de CF se mesure par sa capacité à exclure les fausses filiations. Cette capacité peut être estimée par des calculs de probabilités d'exclusion, lesquelles dépendent des fréquences relatives des allèles observés. Dans le cas d'un système à 2 allèles avec dominance $(\mathrm{A}, \mathrm{a})$ de fréquences respectives $p$ et $q$, la probabilité qu'une erreur de paternité (ou de maternité) soit détectée quand la mère (ou le

\begin{tabular}{|c|c|c|c|}
\hline & & 1ère Famille & 2ème Famille \\
\hline \multirow{2}{*}{ Produit } & $\begin{array}{c}\text { Phénotype } \\
\text { Génotype }\end{array}$ & $\mathrm{AB}$ & $\mathrm{B}$ \\
& Phénotype & $\mathrm{A} / \mathrm{B}$ & $\mathrm{B} / \mathrm{B}$ ou B/- \\
\hline \multirow{2}{*}{ Mère } & Génotype & $\mathrm{A}$ & $\mathrm{B} / \mathrm{B}$ ou B/- \\
& Phénotype & $\mathrm{A} / \mathrm{A}$ ou A/- & $\mathrm{A}$ \\
Père & Génotype & $\mathrm{A}$ & $\mathrm{A} / \mathrm{A}$ ou A/- \\
\hline
\end{tabular}

Tableau 3. Contrôle de I'hérédité des groupes sanguins de deux familles. 
Figure 1.

Schéma d'une électrophorèse en gel

d'amidon.

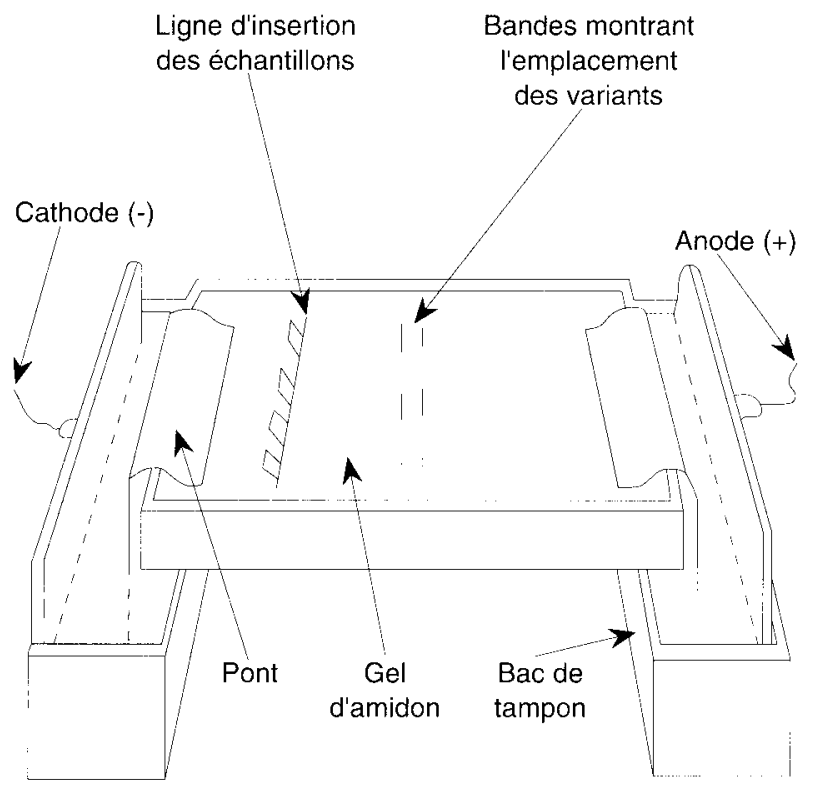

père) est connu(e) avec certitude peut être illustrée de la manière suivante :

enfant $(\mathrm{A} / \mathrm{a})$, mère $(\mathrm{a} / \mathrm{a})$, père présumé $(\mathrm{a} / \mathrm{a})$

La probabilité de rencontre de ce triplet ou pouvoir d'exclusion (Pexcl) de la méthode sera :

Pexcl. $=\underset{(A / a)}{p} \quad x \quad \underset{(a / a)}{q^{2}} \quad x \quad \underset{(a / a)}{q^{2}}=p^{4}$

De même on démontre que pour 1 système à 2 allèles codominants :

Pexcl. $=$ pq $(1-p q)$

La démonstration est généralisable à $\mathrm{N}$ systèmes multialléliques, les probabilités d'exclusion se cumulant. En pratique, la mise en oeuvre d'une dizaine de systèmes génétiques polymorphes permet de tendre asymptotiquement vers une probabilité d'exclusion égale à 1 (Pexcl.=1). Par exemple, le cumul des 12 systèmes génétiques mis en oeuvre chez les ovins conduit à une efficacité de $88 \%$ en race Lacaune.

Le nombre de systèmes utilisés se heurte à des considérations économiques. Il convient donc de trou- ver un équilibre entre le pouvoir d'exclusion et le coût de la méthode, chaque système génétique ayant son propre coût unitaire. Ce coût peut être très variable selon la technique mise en oeuvre : antigènes érythrocytaires, électrophorèse de protéines, antigènes lymphocytaires (systèmes d'histocompatibilité) ou polymorphisme de longueur des fragments de restriction (RFLP) de l'ADN.

Le Laboratoire assure à cet effet une veille technologique sur les techniques nouvelles et notamment sur les recherches menées au Laboratoire de Génétique Biochimique (INRA Jouy-en-Josas) en y détachant du personnel. Les études sur la détection du polymorphisme de l'ADN par RFLP ou par PCR (réaction de polymérisation en chaîne) sont prometteuses et peuvent modifier considérablement les techniques d'analyses dans le but de contrôle des filiations.

\section{Conclusion}

Les recherches sur le polymorphisme génétique des caractères sanguins ont permis de mettre au point, pour les espèces d'élevage, des techniques d'analyses fiables et standardisées (normes internationales). Les résultats des analyses sont introduits dans des fichiers constitués par espèces et par races. Les contrôles de filiation comparent les groupes sanguins des trois membres des familles considérées: père, mère, produit. Le grand nombre de caractères étudiés permet de rendre des conclusions solides détectant la quasi-totalité des fausses filiations. Ces contrôles sont très utilisés par les différents acteurs de l'amélioration génétique des animaux. En effet, la plupart des évaluations génétiques de reproducteurs (indexation) prennent en compte les performances zootechniques d'individus apparentés. Les erreurs de parentés peuvent être estimées par sondage sur des effectifs importants.

En 1991 le Laboratoire de l'INRA a ainsi effectué environ 63000 analyses (toutes espèces confondues) et contrôlé plus de 44000 filiations.

\section{Références bibliographiques}

François D., 1987. Les Groupes Sanguins des bovins. Bovins Limousins, 92, 11-16.

Grosclaude F., Guérin G., Houlier G., 1979. The genetic map of the B system of cattle blood groups as observed in French breeds. Anim Blood Groups Biochem. Genet. 10, 199-212.

Grosclaude F., Aupetit R.Y., Lefèbvre J., Mériaux J.C., 1990. Essai d'analyse des relations génétiques entre les races bovines françaises à l'aide du polymorphisme biochimique. Génét. Sél. Evol., 22, 317-338.

Grosclaude F., François D., Wimitzky M., 1990. Evidence for absence of linkage between the $\mathrm{B}$ and $\mathrm{Z}$, as well as between the $F$ and $S$ systems of cattle blood groups. Anim. Genet. $21,427-429$
Hanset R., 1975. Probabilité d'exclusion de paternité et de monozygotie, probabilité de similitude. Généralisation à N allèles co-dominants. Ann. Med. Vet., 119, 71-80.

Mériaux J.C., 1981. Les groupes sanguins des chevaux et leur utilisation pratique pour l'identification et le contrôle de filiation. B.T.I., 533-548.

Nguyen T.C., 1979. Les groupes sanguins du mouton (Ovis aries). Thèse, Doctorat d'Etat, Université Paris VI, 110p.

Nguyen T.C., 1979. Polymorphisme sanguin du mouton et distances génétique entre races. 5èmes Journées de la recherche Ovine et Caprine, "L'amélioration Génétique des Espèces Ovine et Caprine", Paris, 5 et 6 décembre 1979 , 245-254.

Nguyen T.C., 1990. Genetic systems of red cell blood groups in goats. Anim. Genet., 21, 233-245. 